\title{
Strong bidentate coordination for surface passivation and ligand-shell engineering of lead halide perovskite nanocrystals in the strongly quantum confined regime
}

\author{
Aaron Malinoski, Guoxiang Hu, Chen Wang* \\ Department of Chemistry and Biochemistry, Queens College, City University of New York, 65-30 \\ Kissena Blvd., Flushing, NY 11432 \\ *corresponding author. Email: chen.wang@qc.cuny.edu
}

\begin{abstract}
The surface of lead halide perovskite nanocrystals (PNCs) is unique compared to conventional metal chalcogenide or pnictogenide semiconductor nanoparticles for its ionic character and the dynamic ligand layer, which makes them unstable in stock solutions and hinders the development of surface engineering strategies. This work employs a chelating strategy to form stable coordination on the PNC surface. Through screening a series of heterocyclic aromatic carboxylates, we found the best ligand, picolinate (PIC), with exceptional passivation effect to the surface traps of $\mathrm{CsPbBr}_{3}$ PNCs in the strongly quantum confined regime, resulting in $>0.8$ photoluminescence quantum yields. The exciton lifetime in the passivated PNC approaches the radiative decay limit in various solvents. From an NMR titration experiment, the binding affinity of PIC is estimated to be at least 15 to 30 folds stronger than the original ligand from synthesis. The NMR and FTIR spectroscopic data and first-principles calculations elucidate the bidentate nature of the PIC coordination at the surface $\mathrm{Pb}$ site and the coadsorption of the ammonium-PIC ion pair. In apolar solvents, such as cyclohexane, the binding of PIC is stoichiometric to the available surface sites, suggesting the structure as a potent candidate for anchoring functional molecular structures to the PNC surface. In polar solvents, the strong affinity of PIC on the PNC surface provides protection for carrying out the precipitation-redissolution purification procedure that removes synthetic residual from the as-synthetic PNC samples. By modifying the purification procedure, we also develop a cation exchange procedure to replace the original oleylammonium cation with desired structures that consist of an ammonium anchoring group. Our results provide a direction for constructing strong interactions to protect the vulnerable surface of PNCs and pave the road for developing surface engineering strategies to functionalize these nanoparticles.
\end{abstract}

KEYWORDS: perovskite nanocrystal, chelating, picolinate, surface engineering, passivation 


\section{INTRODUCTION}

Inorganic cesium lead halide perovskites have attracted intense research interest for their promising potentials in applications such as optoelectronics ${ }^{1-3}$ and photocatalysis. ${ }^{4}$ The advances in nanotechnology enable precise control of the $\operatorname{size}^{5}$, shape $e^{6-9}$, and chemical doping ${ }^{10-15}$ of inorganic perovskite nanocrystals (PNCs), providing abundant approaches to tune the optical and electronic properties of this material.

Surface chemistry is one of the most intriguing fields in the research of nanomaterials. For conventional semiconductor nanocrystals, such as metal chalcogenides, their large surfaceto-volume ratio and rich ligand chemistry offer opportunities to mediate the electronic properties and control the exciton behaviors via tailoring the ligand layer. ${ }^{16-22}$ It had been demonstrated that the compact ligand layer could play roles beyond a passive protective shell and act as an active handle to control the accessibility of the semiconductor core to molecules in the environment. ${ }^{23-}$ ${ }^{26}$ Conjugating the nanoparticle and substrates through a designed ligand linker can selectively promote carrier migration. ${ }^{27}$ By introducing proper physical and chemical interactions, it is possible to fabricate hybrid superstructures with nanoparticles and molecules that direct the flow of excitonic energy. ${ }^{28-33}$ Through functionalizing the particle surface, researchers have developed various biosensors based on colloidal quantum dots. ${ }^{34-36}$

PNCs differ from the abovementioned conventional semiconductor nanocrystals because the highly ionic perovskite core is capped with weakly bound, dynamic surface ligands. ${ }^{37-39}$ The vibrant surface delivers opportunities to mediate the shape and dimension of PNCs through modifying the surface ions ${ }^{40-41}$, but more often, the unstable ligand layer leaves a large number of defects and affects the stability of these materials in the solution phase. Although perovskite semiconductors, such as $\mathrm{CsPbBr}_{3}$, are well-known for defect-tolerance ${ }^{42-43}$, when the dimension approaches the strongly quantum confined regime, i.e., small nanocrystals and thin nanoplatelets, 
the loss of excitonic energy becomes severe due to the trapping. ${ }^{44}$ The vulnerable surface also makes it difficult to purify PNCs from the synthetic mixture without losing control of their dimension and surface integrity. ${ }^{45-46}$ Moreover, the lack of stable interaction between the ligand and the perovskite crystal restricts the development of surface engineering strategies for functionalizing PNCs. Although intense research efforts for PNCs have been focused on eliminating surface defects and stabilizing the nanocrystals, ${ }^{47-56}$ until recently, there are only a few reports discussing how surface ligands influence the charge carrier migration from PNCs. ${ }^{57-}$ 59

This paper describes the use of cooperative bidentate coordination to realize a strong affinity to the cesium lead halide PNC surface, which do not only reliably protect the vulnerable surface but also provide opportunities to develop the engineering strategy for modifying the ligand shell. Ligands with multiple binding centers have been reported for improving the surface passivation, and stability of PNCs. ${ }^{50,54}$ We focus on a group of heterocyclic aromatic carboxylates because they integrate two coordination sites that can effectively bind to the $\mathrm{Pb}$ based perovskite surface: the carboxylic group that is connected with aromatic rings as the relatively soft Lewis base ${ }^{47}$, and the heteroatoms that can donate the lone-pair electrons. ${ }^{49,}{ }^{60} \mathrm{We}$ identify a strong ligand, picolinate (PIC), which has a bound affinity to the PNC surface that is 15 to 30 folds stronger than the original ligands and can quantitatively binding to the PNC surface in apolar solvents. The cooperative chelating coordination of PIC at the surface $\mathrm{Pb}$ site is illustrated by NMR and FT-IR spectroscopy and interpreted with first-principle computation. As shown in Figure 1, the strong coordination of the PIC ligand provides effective passivation for surface traps and has the potentials to serve as anchoring motifs for functionalizing the PNC 
surface. We also design a cation exchange procedure based on the strong PIC ligand to introduce new cation ligands to the shell.

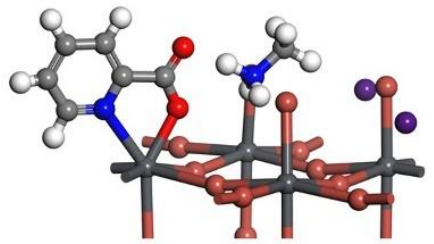

Strong bidentate coordination
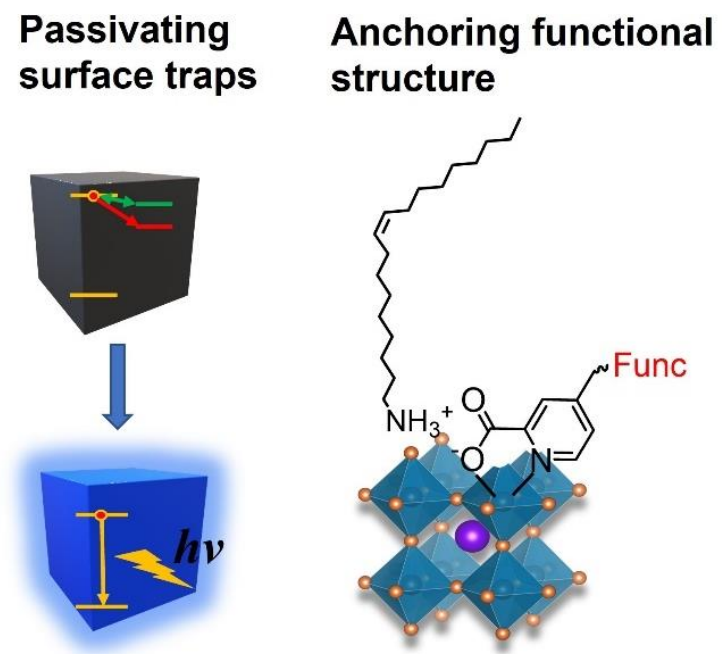

Modifying the cationic ligands

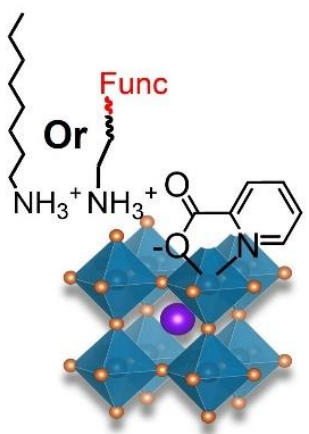

Figure 1. The strongly bound ligand picolinate (PIC) for passivation and modifications of the PNC surface.

\section{RESULTS AND DISCUSSION}

\section{Heterocyclic carboxylate ligands, in particular, picolinate, can effectively protect the} surface of PNCs in various solvents. The PNCs used in the present study are in the strongly quantum confined regime with bandgap energies spanning from $2.51 \mathrm{eV}$ to $2.76 \mathrm{eV}$, as shown in

Figure 2A, corresponding to edge dimensions (d) between $3.7 \mathrm{~nm}$ and $6.6 \mathrm{~nm} .{ }^{61}$ The photoluminescence quantum yield (PLQY) monotonically decreases with the decrease of the particle sizes (Figure 2C) due to the enhanced exciton trapping at surface defects for PNCs in the strongly quantum confined regime (See the Supporting Information). The PLQY of PNCs is also sensitive to solvent polarity, as shown in Figure 2D and reported in earlier work. ${ }^{45-46}$ Polar solvents tend to facilitate the detachment of original ligands from the ionic PNC surface and generate more defect sites that lower the PLQY. ${ }^{51}$ In solvents with high polarities, such as ethyl acetate, the detachment of original ligands is so severe that the exposed defects can induce the fusion of small nanocrystals to large structures with loosened quantum confinement (Figure 
S2 and S3). The vulnerable PNC surface prevents employing the commonly-used precipitationredissolution procedure to purify the as-synthesized PNCs. Therefore, it is an urgent need to find ligands that are firmly bound to the PNC surface for protecting the nanoparticles in various environments.

We studied the passivation effect of various aromatic carboxylates and found that all of them can improve the PLQY of PNCs in toluene, as shown in Figure 2B. Two of them, picolinate (PIC) and 2-thiophene carboxylate (TC), can increase the PLQY of the small PNC (d $=4.3 \mathrm{~nm})$ to $>0.8$. The UV-vis absorption and PL spectra remain unchanged after passivation (Figure S4). The presence of the carboxylic group and the heteroatom site in the adjacent position is critical to achieve the best passivation. When using the single-role carboxylic ligand, benzoate, to passivate the same PNC, 3000 equivalents of ligands are needed to achieve a PLQY of 0.67. Single-role ligands with lone-pair electrons, such as pyridine and thiophene, show little influence on the PLQY of PNCs in the solution phase (See the Supporting Information, Figure S5), though they were reported as effective passivation reagents for improving the performance of photovoltaic devices made of organic-inorganic hybrid perovskites. ${ }^{60}$ Titrating PNCs with a mixture of benzoate and pyridine show a similar passivation effect as $\mathrm{OlAm}^{+} \mathrm{BA}^{-}$, which is less effective compared to the heterocyclic carboxylate ligands (Figure S5). Finally, isonicotinate, the para-pattern isomer of picolinate, shows poor passivation among all ligands.

The passivation of PIC is exceptionally effective: with 400 equivalents of addition to the PNC with $\mathrm{d}=4.3 \mathrm{~nm}$ (corresponding to 3.6 PIC per $\mathrm{nm}^{2}$ of the PNC surface), the sample reaches the maximum PLQY of 0.81 . The amount of PIC to achieve the optimal passivation is only $25 \%$ surpass the total number of $\mathrm{Pb}$ atoms on the surface layer of perovskite crystal, suggesting its strong surface affinity. The decline of PL intensity after 400 equivalents is caused by the 
degradation of PNCs in the presence of extra PIC.(See the Supporting Information, Figure S6, and S7.) We speculate that at high concentration, the extra amount of PIC anion can etch the PNC by stripping off the surface $\mathrm{Pb}$ to form neutral $\mathrm{Pb}(\mathrm{PIC})_{2}$ complexes that are easier to dissolve in the solution phase. The PIC passivation increases the PLQYs of PNCs with all sizes and in various solvents, as shown in Figure 2C and D. The improvement of PLQY is more prominent for samples that originally have a low PL such as small-size PNCs and PNCs in relatively polar solvents. Strikingly, the PIC can prevent the fusion of small-size PNCs in polar solvents, such as ethyl acetate and dimethoxyethane, in which PNCs with the native ligands have poor solubility. (See the Supporting Information, Figure S2, and S3) 

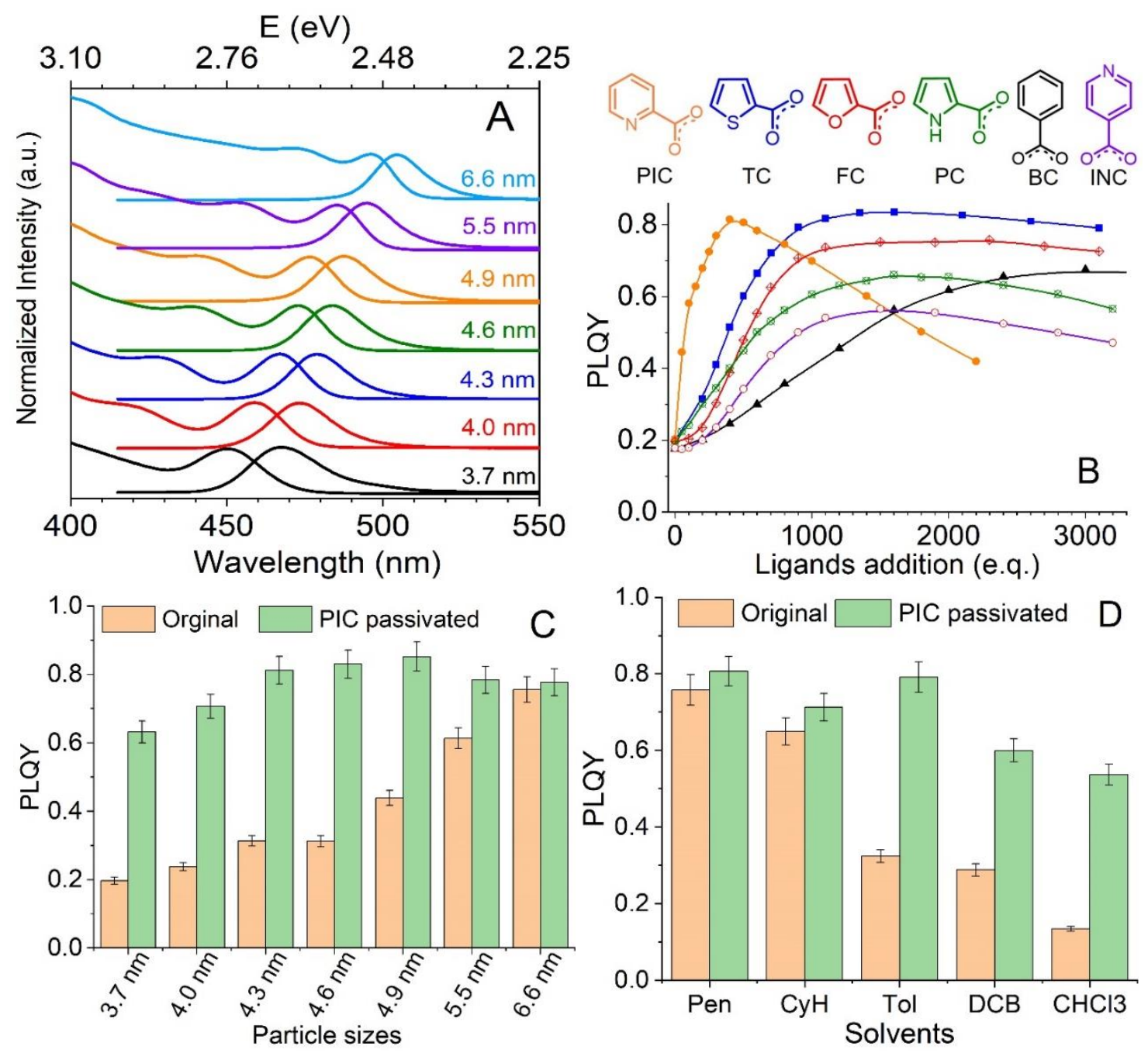

Figure 2. (A) Normalized UV-vis absorption and photoluminescence spectra in toluene. The sizes of PNCs are listed in the graph. (B) The change of PLQYs of the PNC with $\mathrm{d}=4.3 \mathrm{~nm}$ in toluene with the addition of six different passivation ligands: benzoate (BC), thiophene-2carboxylate (TC), Furan-2-carboxylate (FC), pyrrole-2-carboxylate (PC), picolinate (PIC), and isonicotinate (INC). The amounts of titration ligands are converted to equivalents per PNC. The lines are employed to follow the trend of changes. Molecular structures of the ligands are shown above the graph and coded with the same colors as the PLQY traces. The concentration of the PNC solution is $0.05 \mu \mathrm{M}$. Ligands were titrated as their oleylammonium $\left(\mathrm{OlAm}^{+}\right)$salts to the PNC sample dissolved in toluene. PLQYs were determined with $405 \mathrm{~nm}$ excitation. (C) PLQYs of PNCs with different sizes in toluene before and after PIC passivation. All samples have an optical density of 0.22 at $405 \mathrm{~nm}$. Note that the PLQY here for the unpassivated, $\mathrm{d}=4.3 \mathrm{~nm}$ PNC is higher than the value shown in (B) because we had to use a lower concentration in the titration experiment. For PNCs that are capped with weakly bound original ligands, their PLQY depends on the concentration. ${ }^{47}$ (D) PLQYs of PNCs with $\mathrm{d}=4.3 \mathrm{~nm}$ in various solvents before and after PIC passivation. Abbreviations of the solvents: Pen: pentane, $\mathrm{CyH}$ : cyclohexane, Tol: toluene, DCB: 1,2-dichlorobenzene, and $\mathrm{CHCl}_{3}$ : chloroform. 
Time-resolved photoluminescence (TR-PL) kinetics (Figure 3A and Figure S8) illustrate that PNC samples consist of two subensembles with distinct exciton decay lifetimes: a fast-decay ensemble that relaxes rapidly at a rate faster than the instrument response $(<0.57 \mathrm{~ns})$ representing the "dark" population of PNCs, and a slowly-decay ensemble featuring observable time constants of 3.2 - $6.2 \mathrm{~ns}$ representing the "bright" population. The inhomogeneous emission is common for semiconductor nanocrystal samples, including PNCs. ${ }^{62-63}$ The PIC passivation has two effects: increasing the population of the "bright" and elongating the observable exciton lifetimes, in which the former is the major contribution to the enhancement of PL in solvents such as toluene, $\mathrm{DCB}$, and $\mathrm{CHCl}_{3}$. For instance, in toluene, the integrated intensity of the longlifetime component increases by 2.2 folds upon passivation, while the observed lifetime before and after passivation only elongates from 3.5 to $4.7 \mathrm{~ns}$. In comparison, the steady-state experiment measures a 2.5-fold increase for the PLQY. The initial fast-decay component remains in the kinetics for all passivated samples, suggesting that a subgroup of the "dark" PNCs possess defects that cannot be fully removed with PIC passivation, leading to the less-than-unity PLQY after PIC passivation. 

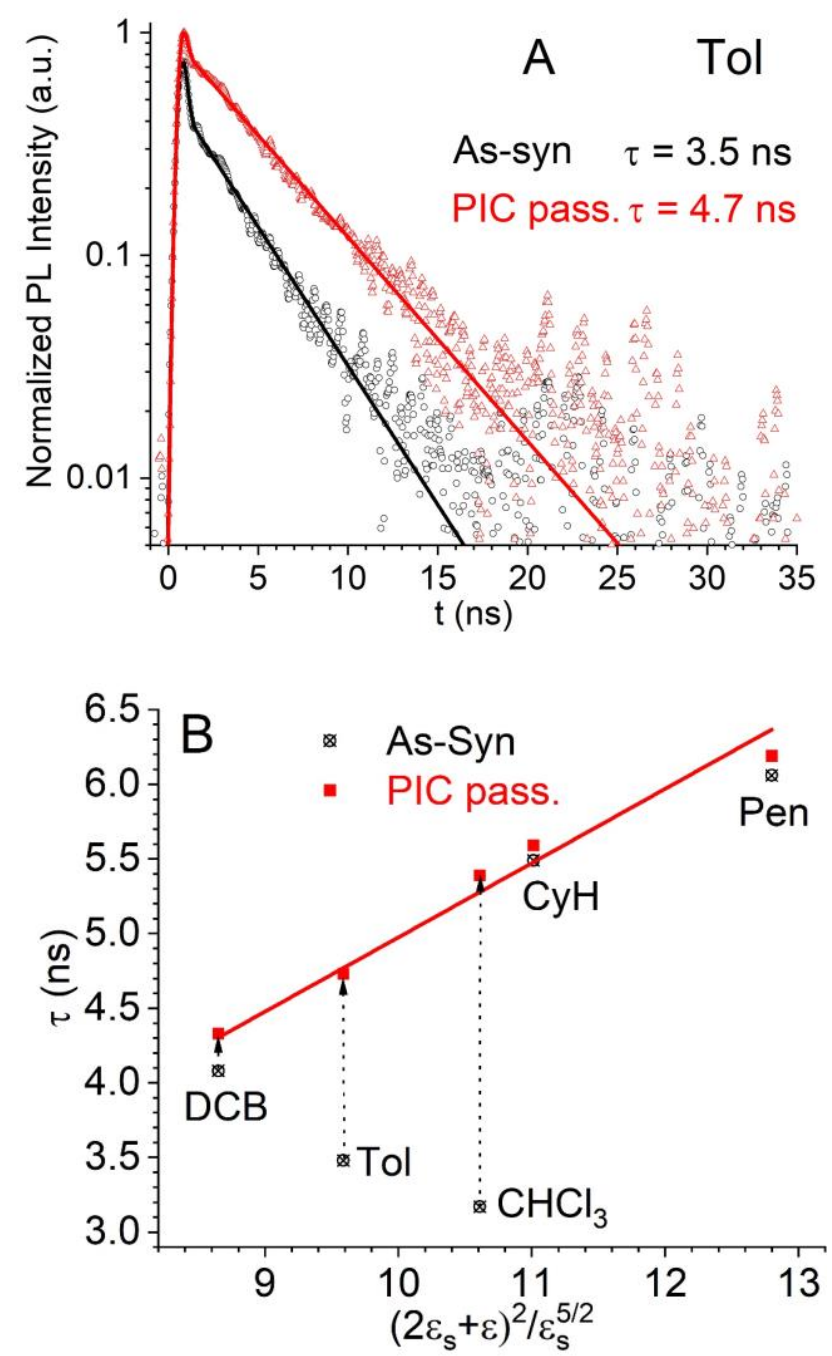

Figure 3. (A) Time-resolved PL kinetics of PNCs with $\mathrm{d}=4.3 \mathrm{~nm}$ before (black) and after (red) passivation with PIC recorded in toluene. The kinetics traces of the passivated sample are recorded when the highest PL intensity is achieved by titrating $\mathrm{OlAm}^{+} \mathrm{PIC}^{-}$ligands. The PL intensity is normalized to the maximum of the passivated sample. The kinetic traces are fit to a convoluted function of the IRS function $(\mathrm{FWHM}=0.57 \mathrm{~ns})$ and a double-exponential decay. We determine that an exponential component with a time constant that is significantly shorter than the IRS is necessary to yield the best fit of the initial fast decay, but it has no physical meaning because it is beyond the limit of our instrument. The observable lifetimes from the fit of the longlived decay are listed in the figure. PL kinetics of PNCs in other solvents are shown in the Supporting Information. Lifetimes of the passivated sample in different solvents are plotted as the function of the dielectric factor, $\frac{\left(\varepsilon+2 \varepsilon_{s}\right)^{2}}{\varepsilon_{s}^{5 / 2}}$, in $(\mathbf{B})$. The red line shows the linear fit for the lifetimes of the passivated samples in different solvents.

For the passivated PNC sample, the measurable exciton lifetimes monotonically decrease with the increase of the solvent optical dielectric constants. In contrast, before passivation, the lifetimes have no apparent correlations with the solvent properties. The radiative lifetime of a 
transition dipole within the nanoparticle is proportional to the factor of $\frac{\left(2 \varepsilon_{S}+\varepsilon\right)^{2}}{\varepsilon_{S}^{5 / 2}}$, when considering the dielectric screening effect of the radiating field inside the nanoparticles, ${ }^{64-65}$ where $\varepsilon_{s}$ is the optical dielectric constants of solvents and $\varepsilon \approx 4$ for $\mathrm{CsPbBr}_{3}$ in the pertained spectral window. ${ }^{66}$ Our measurements of the passivated sample show a good linear relationship when plotted against the dielectric factors (Figure 3B), suggesting that exciton lifetimes in the passivated PNC proximate the radiative limit of PNCs. These lifetimes are indeed very close to the reported lifetimes of PNCs with near-unity PLQY ${ }^{47-48}$, implying that after PIC passivation, PNCs in the "bright" subensemble are nearly trap-free. Our observation also provides evidence for the close contact between perovskite cores and solvent molecules, as it has been suggested for the PNCs with original weakly bound ligands. ${ }^{61}$

We summarize the passivation of the PNCs in Scheme 1, with the consideration of exciton traps with different energy levels: the shallow trap that causes the slow non-radiative decay in the nanosecond regime, and the deep trap that is responsible for the ultrafast PL decay beyond the instrument response. These traps are likely induced by different defects, and their distributions in PNCs are affected by solvent properties, as suggested by the PL kinetics (See the Supporting Information). Without passivation, the "bright" subensemble of PNCs must be free of deep traps, which otherwise overrun the radiative process. For these "bright" PNCs, the PIC passivation removes shallow traps and enhances their emission. The more prominent effect of the PIC passivation lies in the elimination of a large portion of defects that induce deep traps, which transforms the original "dark" PNCs to bright. The remaining of a small subemsemble of "dark" PNC with an ultrafast PL decay after passivation is similar to persistent traps observed for perovskite nanoplatelets. ${ }^{62} \mathrm{We}$ speculate that these PNCs may have defects inside the crystal that are not accessible by the surface ligands. Another possibility is that due to the relatively large 
steric hindrance of the PIC ligands, they cannot fill in all surface sites and leave some particles with partially passivated surfaces. A more detailed investigation on the properties of different types of defects and their interactions with the passivating ligands and the surrounding is currently being carried out in our group.

Scheme 1. The PIC passivation effect on PNC subensembles with different defects

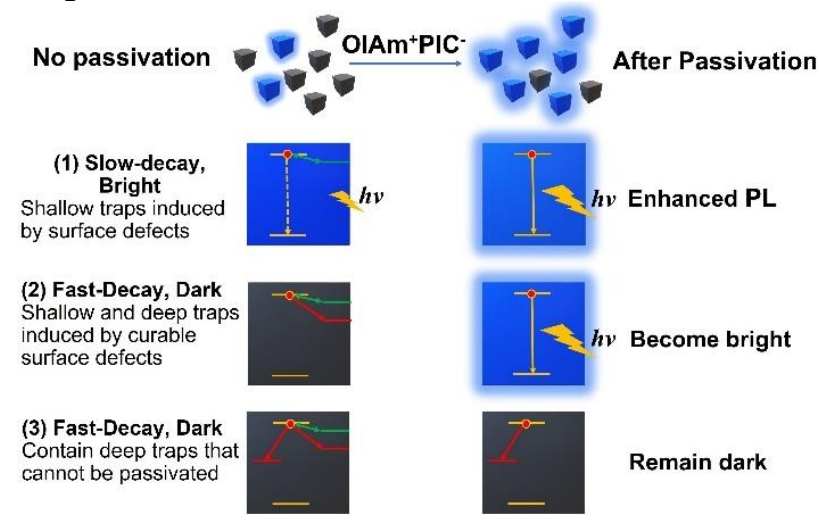

\section{The excellent passivation/protection effect results from the strong bidentate}

coordination of PIC to the PNC surface. To evaluate the bound affinity of PIC, we designed an NMR titration experiment. We added a series of known amounts of $\mathrm{OlAm}^{+} \mathrm{PIC}^{-}$ligands to the solution of as-synthesized PNCs, as shown in Figure 4A. The concentration of $\mathrm{OlAm}^{+} \mathrm{PIC}^{-}$was kept in the range that the decomposition of $\mathrm{PNCs}$ due to the possible formation of $\mathrm{Pb}(\mathrm{PIC})_{2}$ complex was neglectable, as demonstrated in the UV-vis absorption spectra (Figure S6). Severe broadening of ${ }^{1} \mathrm{H}$ resonance signals is observed for PIC at 8.4-8.7 and 8.1-8.3 ppm. The broadened NMR signals imply the association of PIC to the nanoparticle surface, which restricts the tumbling and leads to more efficient transversal relaxation, caused by dipolar couplings. ${ }^{67-68}$ The broadening effect is more prominent for the signal of ${ }^{1} \mathrm{H}(\mathrm{I}, 8.4 \sim 8.7 \mathrm{ppm})$ than that of ${ }^{1} \mathrm{H}(\mathrm{II}$, 8.1 8.3 ppm), suggesting the former is closer to the PNC surface when bound. ${ }^{68}$ Since ${ }^{1} \mathrm{H}(\mathrm{I})$ and ${ }^{1} \mathrm{H}(\mathrm{II})$ are located at the opposite positions relative to the pyridine $\mathrm{N}$ atom, the distinct 
broadening effects illustrate the proximity of the nitrogen to the PNC surface, which implies the participation of the pyridine in coordination with the surface site.

Moreover, the integrated intensities of PIC NMR signals are significantly lower than the expected values based on the addition, as listed in Table 1. It is known for small and rigid ligands that the broadening can be so severe that their NMR signals are obscured when they are tightly bound to the nanoparticle surface ${ }^{69} \mathrm{We}$, therefore, assign the disappeared PIC signals to the tightly bound ligands, while the observed signals to PIC that is dispersed in the solution and undergoes fast exchange.

With this assumption, we model the competitive adsorption between $\mathrm{OlAm}^{+} \mathrm{PIC}^{-}$and the coexisted original ligands $\mathrm{OlAm}^{+} \mathrm{Br}^{-}$(notated as L) with Langmuir isotherms:

$$
\begin{aligned}
& \theta_{L}=\frac{N_{L}}{N_{\text {total }}}=\frac{K_{e q}^{L}[L]}{1+K_{e q}^{L}[L]+K_{e q}^{P I C}[P I C]} \\
& \theta_{P I C}=\frac{N_{P I C}}{N_{\text {total }}}=\frac{K_{e q}^{P I C}[P I C]}{1+K_{e q}^{L}[L]+K_{e q}^{P I C}[P I C]}
\end{aligned}
$$

in which $K_{e q}^{L}=10.5 \mathrm{mM}^{-1}$, is the bound equilibrium constant for the original ligand. ${ }^{47}[\mathrm{~L}]$ is the concentration of original ligands in the solution phase, and $[\mathrm{L}]=\mathrm{C}_{L}-\theta_{L} C_{P N C} N_{\text {total }} . C_{P N C}=2$ $\mu \mathrm{M}$, is the concentration of the $\mathrm{PNC}$, and $\mathrm{C}_{L}=2.12 \mathrm{mM}$ is total concentrations of the original ligand determined by NMR for the sample. $N_{\text {total }}$ is the maximum number of potential $\mathrm{Pb}$ sites on one nanocrystal, given by $N_{\text {total }}=6\left(\frac{d}{a}\right)^{2}$, in which $a=0.587 \mathrm{~nm}$ is the dimension of the unit cell and $d=4.3 \mathrm{~nm}$. [PIC] is the concentration of PIC dispersed in the solution, obtained by comparing the integrated NMR intensity with the internal standard, and $N_{P I C}$ is the equivalent number of the disappeared PIC. In this model, the competitive adsorption from oleate ( $\left.\mathrm{OA}^{-}\right)$is not included because $\mathrm{OA}^{-}$is known as a weaker ligand compared to $\mathrm{OlAm}^{+} \mathrm{Br}^{-37,47}$, and we estimate the bound affinity of OA-is only 1/250 compared to that of PIC (See the Supporting 
Information). It also needs to point out that the model does not consider the inhomogeneity of surface sites.

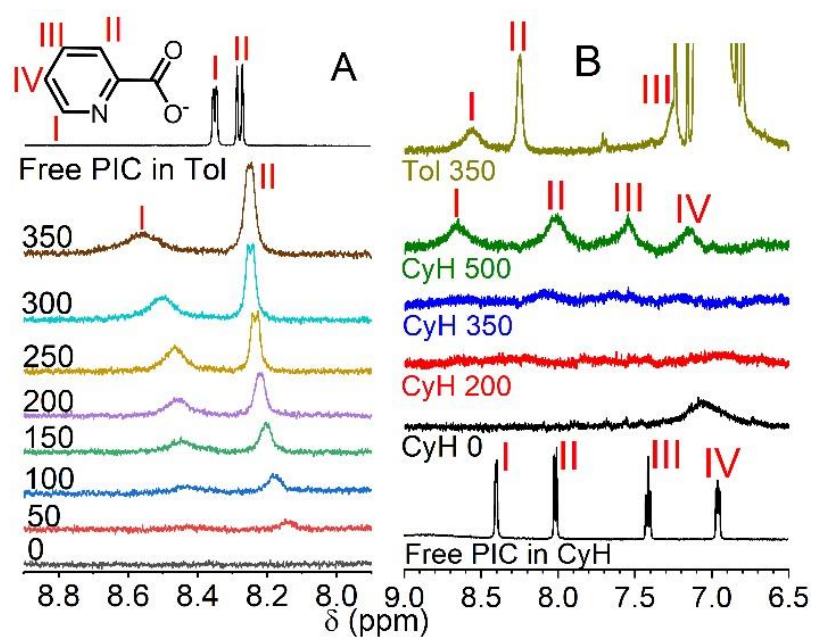

Figure 4. The evolution of ${ }^{1} \mathrm{HNMR}$ signals of PIC when titrating different amounts of $\mathrm{OlAm}^{+} \mathrm{PIC}$-ligands to the PNC solutions of (A) toluene-D8 (B) cyclohexane-D12. The number of observed PIC ligands per PNC is determined by comparing integrated peak intensities in (A) with that of the internal standard, and the results are listed in Table 1. The molecular structure of PIC is shown to illustrate the ${ }^{1} \mathrm{H}$ positions. A spectrum with 350 equivalents addition of PIC in the toluene-D8 solution of PNCs is shown in (B) on the same scale for comparison.

With Eq. 1 and 2, we solve for the bound equilibrium constant, $K_{e q}^{P I C}$, for each PIC titration point, and the results are listed in Table 1. From the different intensities of the ${ }^{1} \mathrm{H}(\mathrm{I})$ and ${ }^{1} \mathrm{H}(\mathrm{II})$ signals, we obtained average values of $K_{e q}^{P I C}=310 \pm 60 \mathrm{mM}^{-1}$ and $150 \pm 20 \mathrm{mM}^{-1}$, respectively. The affinity of PIC is thus $15 \sim 30$ folds greater than that of the original ligand, $\mathrm{OlAm}^{+} \mathrm{Br}^{-}$.

Table 1 Bound and unbound PIC at different titration points and $K_{e q}^{P I C}$ calculated from the NMR data using Eq. 1 and $\mathbf{2}$

\begin{tabular}{ccccc}
\hline $\begin{array}{c}\text { Titration } \\
\text { (per PNC) }\end{array}$ & $\begin{array}{c}{[\mathrm{PIC}] \mathrm{H}(\mathrm{I})} \\
(\text { per PNC) }\end{array}$ & $\begin{array}{c}K_{e q}^{P I C}\left(\mathrm{mM}^{-1}\right) \\
\text { based on H(I) }\end{array}$ & $\begin{array}{c}{[\mathrm{PIC}] \mathrm{H}(\mathrm{II})^{2}} \\
\text { (per PNC) }\end{array}$ & $\begin{array}{c}K_{e q}^{P I C}\left(\mathrm{mM}^{-1}\right) \\
\text { based on H(II) }\end{array}$ \\
\hline 100 & 12 & 329 & 22 & 136 \\
150 & 24 & 262 & 38 & 136 \\
200 & 40 & 250 & 60 & 127 \\
250 & 57 & 273 & 74 & 167 \\
300 & 77 & 313 & 100 & 172 \\
350 & 96 & 427 & 135 & 158 \\
\hline Average & -- & $310 \pm 60$ & -- & $150 \pm 20$ \\
\hline
\end{tabular}

1 determined based on the integrated NMR intensity of ${ }^{1} \mathrm{H}(\mathrm{I}): 8.4 \sim 8.7 \mathrm{ppm}$.

${ }^{2}$ determined based on the integrated NMR intensity of ${ }^{1} \mathrm{H}(\mathrm{II}): 8.1 \sim 8.3 \mathrm{ppm}$. 
In the less polar solvent, cyclohexane, the broadened PIC signals only become prominent when $>350$ equivalents of ligands are added (Figure 4B). Though it is unlikely that the bulky PIC ligands can form a dense coverage of $3.2 \mathrm{~nm}^{-2}$ (given all 350 PIC are tightly bound to the surface), the absence of the NMR signal suggests the strong association of $\mathrm{OlAm}^{+} \mathrm{PIC}^{-}$ion pairs to the PNC surface in the apolar environment. At relatively low ligand loadings $(<200$ equivalents), we believe that PIC can achieve a close-to-stoichiometric bound to the available surface site, and therefore can be employed as the anchoring group to graft functional structures to the PNC surface, provided its aromatic ring can be decorated.

To further understand the binding of the ligands on PNC, we performed ab initio density functional theory (DFT) simulations. We find that the stabilization energy of PIC as a bidentate ligand is prominently lower than the monodentate counterparts. (Figure 5, and Figure S13) The theoretical prediction agrees with the results of the passivation experiments, in which single-role ligands such as isonicotinate, benzoate, and pyridine all show poorer passivation for the PNC surface. When PIC binds to the PNC surface, the $\mathrm{O}$ atom of the carboxylic group and the $\mathrm{N}$ atom of the pyridinic ring coordinate to the undercoordinated $\mathrm{Pb}$ site cooperatively, as shown in Figure 5B, which gains extra stabilization energy of $0.31 \mathrm{eV}$ compared to the single-role binding mode through the carboxylic group. This binding configuration predicted by DFT calculations agrees with the ${ }^{1} \mathrm{HNMR}$ observation that suggests proximation of the $\mathrm{N}$ atom of PIC to the surface. The chelating coordination with two distinct atoms covalently interacted with the same surface $\mathrm{Pb}$ site differs from previously reported bidentate ligands and zwitterion ligands for PNCs . ${ }^{50,53-54}$ 


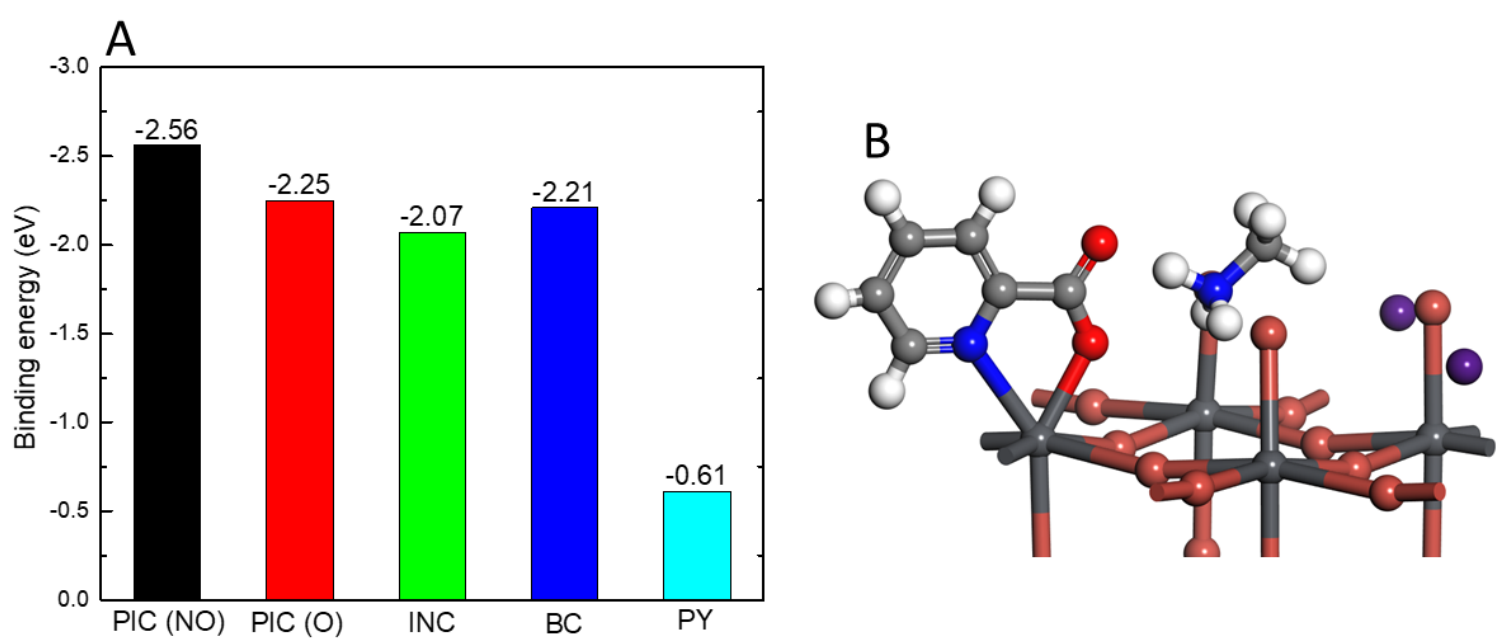

Figure 5. (A) Calculated binding energies (eV) of the ligands on PNC and (B) the optimized binding configuration of PIC on the PNC surface. $\mathrm{H}$, white; $\mathrm{C}$, grey; $\mathrm{O}$, red; $\mathrm{N}$, blue; Cs, purple; $\mathrm{Br}$, brown; $\mathrm{Pb}$, dark grey.

The strong interaction of PIC on the PNC surface enables thorough purification of as-synthesized PNCs and modification of the ligand shell. We developed a precipitationredissolution purification strategy for PNCs based on the effective protection of PIC in ethyl acetate. Typically, 10 nanomoles of as-synthesized PNCs were dispersed in a $10 \mathrm{ml}$ solution of ethyl acetate containing a mixture of $0.7 \mathrm{mM}$ picolinic acid and $1.4 \mathrm{mM}$ OlAm. The turbid suspension was sonicated for $30 \mathrm{~min}$ and centrifuged at $3500 \mathrm{rpm}$ for $5 \mathrm{~min}$ at $4{ }^{\circ} \mathrm{C}$ to recover the PNCs. The recovered PNC was redissolved in toluene or cyclohexane, and insoluble solids are removed by centrifugation. The UV-vis absorption and the PL spectra (Figure 6A) of the purified PNC are narrower, with reduced intensity on the blue side of the first exciton band, likely because smaller-size PNCs are harder to precipitate from ethyl acetate. The recovery of PNCs after one round of purification is typical $>0.85$. The purification step can be repeated to further eliminate the synthetic residue, but the recovery decreases to $\sim 0.20$ due to the poor solubility of PNCs after the second ethyl acetate wash. The PLQY of PNCs after the first and second purification is 0.32 and 0.54 , respectively. For the one-round purified sample, the PLQY can be increased to 0.75 by adding another 200 equivalents of $\mathrm{OlAm}^{+} \mathrm{PIC}^{-}$during the 
redissolving step. The insoluble PNCs after the second purification remain quantum-confined. Using a procedure developed by Sargent and coauthors ${ }^{46}$, we fabricated a solid-state film with PNCs after two rounds of purification. As shown in Figure 6A, the PNC film demonstrates a similar PL spectrum as in the solution phase, suggesting PIC can protect the nanocrystals from fusion in the solid.

$>97 \%$ of the residual synthetic solvent, ODE (from $4000 \pm 400$ to $<100$ per PNC), can be removed with one round of purification, and ODE can be fully removed after repeating the procedure. The residual $\mathrm{OA}^{-}$and $\mathrm{OlAm}^{+}$from synthesis decrease from $5000 \pm 600$ to $350 \pm 60$ per PNC, corresponding to a surface coverage of $3.2 \pm 0.6 \mathrm{~nm}^{-2}$, and $200 \pm 40$ per PNC $(1.8 \pm 0.4$ $\mathrm{nm}^{-2}$ ) after the first and second round of purification, respectively. For the insoluble PNCs, the coverage of the long-chain ligands is only $\sim 0.7 \mathrm{~nm}^{-2}$. We, therefore, attribute the poor solubility of samples after two rounds of purification to the loss of long-chain surface ligands during the ethyl acetate wash. 


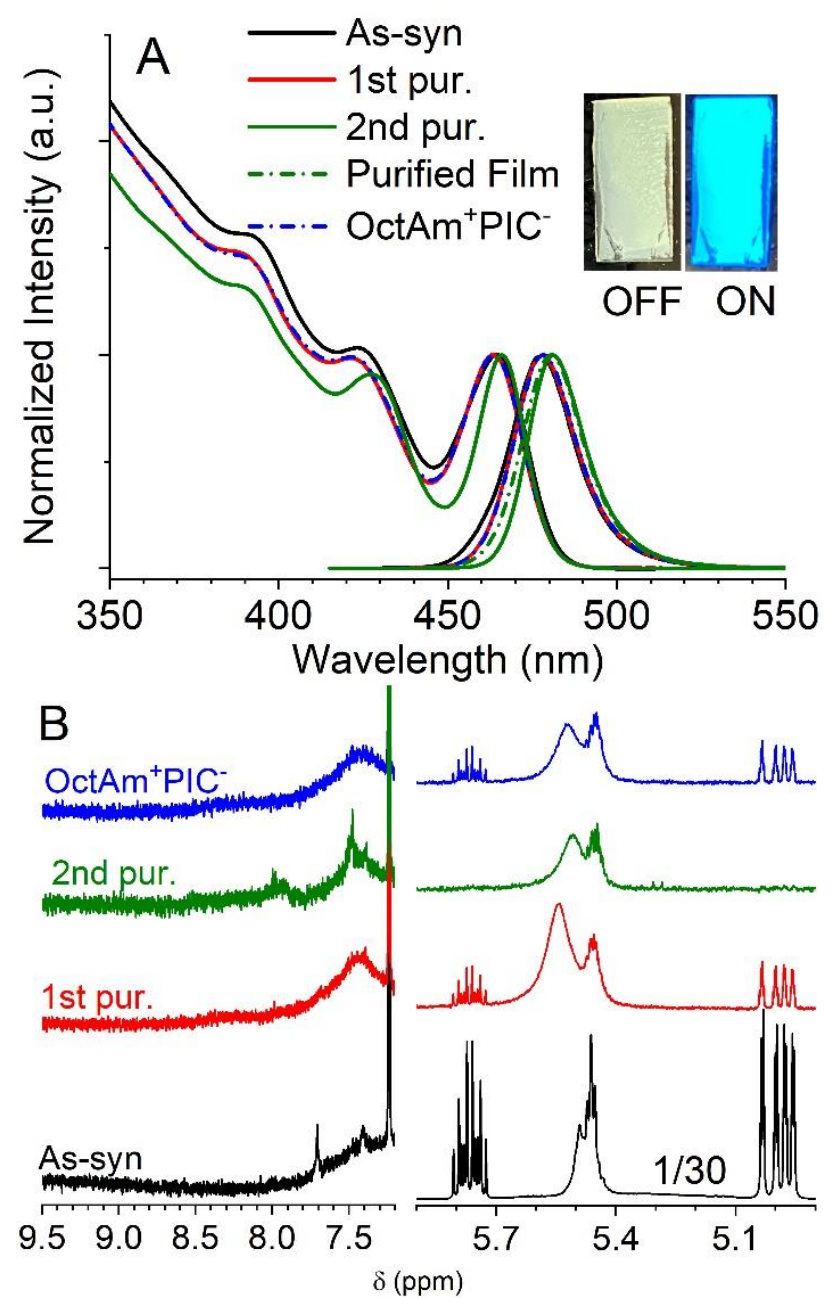

Figure 6. Comparison of PNC samples $(\mathrm{d}=4.3 \mathrm{~nm})$ before and after the first and second purification with $\mathrm{OlAm}^{+} \mathrm{PIC}^{-}$and the cation exchange treatment with $\mathrm{OctAm}^{+} \mathrm{PIC}^{-}$. (A) UV-vis absorption and the PL spectra. The PL spectrum of the film made of PNCs after the two rounds of purification is shown. Inset: images of the film with and without the $365 \mathrm{~nm}$ UV illumination. (B) NMR in toluene-D8. Right: the high chemical shift region and Left: the alkene resonance region. The spectral intensity is normalized according to the intensity of the internal standard and adjusted to the same PNC concentration. The spectrum of the as-synthesized PNC in the right panel is scaled down to its $1 / 30$ so that the intensities are comparable to the purified samples.

We employ ${ }^{1} \mathrm{H}-\mathrm{NMR}$ spectroscopy to characterize the surface ligands of the assynthesized and the purified PNC (Figure 6B). The remained original ligands $\left(\mathrm{OA}^{-}\right.$and $\left.\mathrm{OlAm}^{+}\right)$ show two distinct NMR features in the alkene resonance region. We assigned the broad resonance band above $5.5 \mathrm{ppm}$ to $\mathrm{OlAm}^{+}$and the relatively sharp multiplet at $5.46 \mathrm{ppm}$ to oleate, according to the result of a titration experiment (See the Supporting Information). The former accounts for $>80 \%$ of the long-chain ligands after purification. Surprisingly, NMR observes 
nearly no ${ }^{1} \mathrm{H}$ resonance from PIC for the purified samples. After completely decomposing the purified PNC in deuterated DMSO, we quantify that there are $30 \pm 5$ equivalents of PIC per PNC for samples after the first purification, and $90 \pm 30$ equivalents for samples after the second purification, corresponding to PIC coverage of $0.27 \pm 0.05$ and $0.8 \pm 0.3 \mathrm{~nm}^{-2}$, respectively. The absence of PIC signals in the NMR spectrum in toluene suggests that all PIC ligands are tightly bound to the PNC surface when there is a lack of competitive adsorption from the remained extra synthetic ligand. We, therefore, believe if one decorates the aromatic ring of PIC with desired chemical structure, the PIC motif can serve the role of anchoring groups for grafting functional chemicals to the PNC ligand shell.

FTIR spectroscopy confirms the result of purification (Figure S14). The IR signal of the as-synthesized PNC is a combination of absorption from ODE and the $\mathrm{OlAm}^{+} \mathrm{OA}^{-}$ligand. After purification, the sharp ODE signals at 991 and $908 \mathrm{~cm}^{-1}$ disappear from the spectrum. FTIR also captures notable PIC signals despite the absence of the PIC NMR signals. These unique vibrational features provide important information for illustrating the detailed binding configuration of PIC and will be discussed in a later section.

By comparing the surface components of PNC samples after the first and second purification, we notice that the loss of $\mathrm{OlAm}^{+}$cations (-120 equivalents per $\mathrm{PNC}$ ) is greater than the increase of $\mathrm{PIC}^{-}$anion (60 e.q. per PNC) after the second purification step. The unbalanced component change implies that when the ethyl acetate treatment rips off $\mathrm{OlAm}^{+} \mathrm{Br}^{-}$from the PNC surface, the bulkier PIC- anion cannot fill all $\mathrm{Br}$ vacancies. The downshift of $\mathrm{OlAm}^{+}$alkene resonance from 5.54 to $5.51 \mathrm{ppm}$ after the second purification also indicates the relative weaker association of $\mathrm{OlAm}^{+}$to the PNC surface, likely due to the decrease of the negatively charged hydrogen bond acceptors. 
The purification procedure can be adapted to a cation exchange process for replacing the original $\mathrm{OlAm}^{+}$with other cationic ligands. As a demonstration, we substituted oleylamine in the ethyl acetate washing solution with short-chain octylamine (OctAm), while the rest of the procedure remains the same. The UV-vis absorption and the PL spectra of the resulted sample are identical to the purified PNCs (Figure 6A). With one round of cation exchange, the total number of $\mathrm{OlAm}^{+}$reduces to 170 per PNC, $40 \%$ smaller than the OlAm-treated sample, according to the alkene ${ }^{1} \mathrm{H}$ resonance in the NMR spectrum (Figure 6B). Although ${ }^{1} \mathrm{HNMR}$ cannot distinguish OctAm ${ }^{+}$, the replacement of $\mathrm{OlAm}^{+}$with $\mathrm{OctAm}^{+}$is supported by the downshift of the $\mathrm{OlAm}^{+}$from 5.54 to $5.51 \mathrm{ppm}$, compared to the OlAm-treated sample. We attribute the downshift to a more dynamic exchange of $\mathrm{OlAm}^{+}$on and off the PNC surface due to the competition from $\mathrm{OctAm}^{+}$. The shorter surface ligand can reduce the insulation of the

nanocrystal surface and hence facilitate carrier transportation. ${ }^{70-72}$ It is also possible to employ the cation exchange procedure to introduce functional organic molecules to the surface of PNCs. For example, organic semiconductors have been anchored to the surface of hybrid perovskite materials as ammonium-associated ligands. ${ }^{73-74}$ Our method can be employed to realize similar functionalization for all-inorganic colloidal PNCs.

NMR and IR characterization of the purified sample reveals the chelating configuration and the coadsorption of PIC-ammonium ion pairs. With 200 equivalents of OlAm ${ }^{+} \mathrm{PIC}^{-}$added to the purified PNC sample, 1D- ${ }^{1} \mathrm{HNMR}$ captures two broadened ${ }^{1} \mathrm{H}$ resonance signals of PIC in toluene-D8 at $8.95\left({ }^{1} \mathrm{H}(\mathrm{I})\right)$ and $8.18 \mathrm{ppm}\left({ }^{1} \mathrm{H}(\mathrm{II})\right)$, and the alkene resonance of $\mathrm{OlAm}^{+}$downshifted to $5.50 \mathrm{ppm}$, indicating faster exchange on and off the surface for $\mathrm{OlAm}^{+}$when the total amount of amine increases. (Figure 7A). The 2D NOESY experiment identifies negative NOE at the off-diagonal cross-peaks for bound $\mathrm{OlAm}^{+}$and PIC, as shown in 
Figure 7B (and magnified in Figure S12). The negative NOE confirms the binding of these ligands. ${ }^{68}$ In the alkene proton resonance region, we found that the broad resonance at $5.50 \mathrm{ppm}$ shows clear off-diagonal cross-peaks with negative NOE. In contrast, the NOE effect associated with the $5.46 \mathrm{ppm}$ shoulder is nearly null. The distinction in NOESY confirms the assignments of the former signal to the surface-associated $\mathrm{OlAm}^{+}$and the latter to the weakly bound $\mathrm{OA}^{-}$. For PIC, the negative NOE uncovered the resonance signals of ${ }^{1} \mathrm{H}(\mathrm{III})$ and ${ }^{1} \mathrm{H}(\mathrm{IV})$ at 7.17 and 6.86 ppm, respectively, which are buried underneath the strong solvent (toluene) residual signals in the $1 \mathrm{D}$ spectrum. Moreover, we identify NOE signals belonging to the intermolecular coupling between PIC and OlAm. The NOE associated with the ${ }^{1} \mathrm{H}(\mathrm{II})$ signal of PIC at $8.18 \mathrm{ppm}$ and the ${ }^{1} \mathrm{H}(2)$ of $\mathrm{OlAm}$ at $1.86 \mathrm{ppm}$ provides evidence $\mathrm{OlAm}^{+}$and $\mathrm{PIC}^{-}$are adsorbed on the $\mathrm{PNC}$ surface as cation-anion pairs. The coadsorption of $\mathrm{OlAm}^{+} \mathrm{PIC}^{-}$pair is also supported by our observation while optimizing the purification procedure: when reducing the PIC from 700 to 350 equivalents and using the same 1400 equivalents of OlAm, after crushing the PNC with ethyl acetate, the precipitated PNC is hardly soluble in toluene, likely due to inadequate coverage of the longchain ammonium ligand. This phenomenon suggests that it is the PIC anion that assists the adsorption of the ammonium species. 


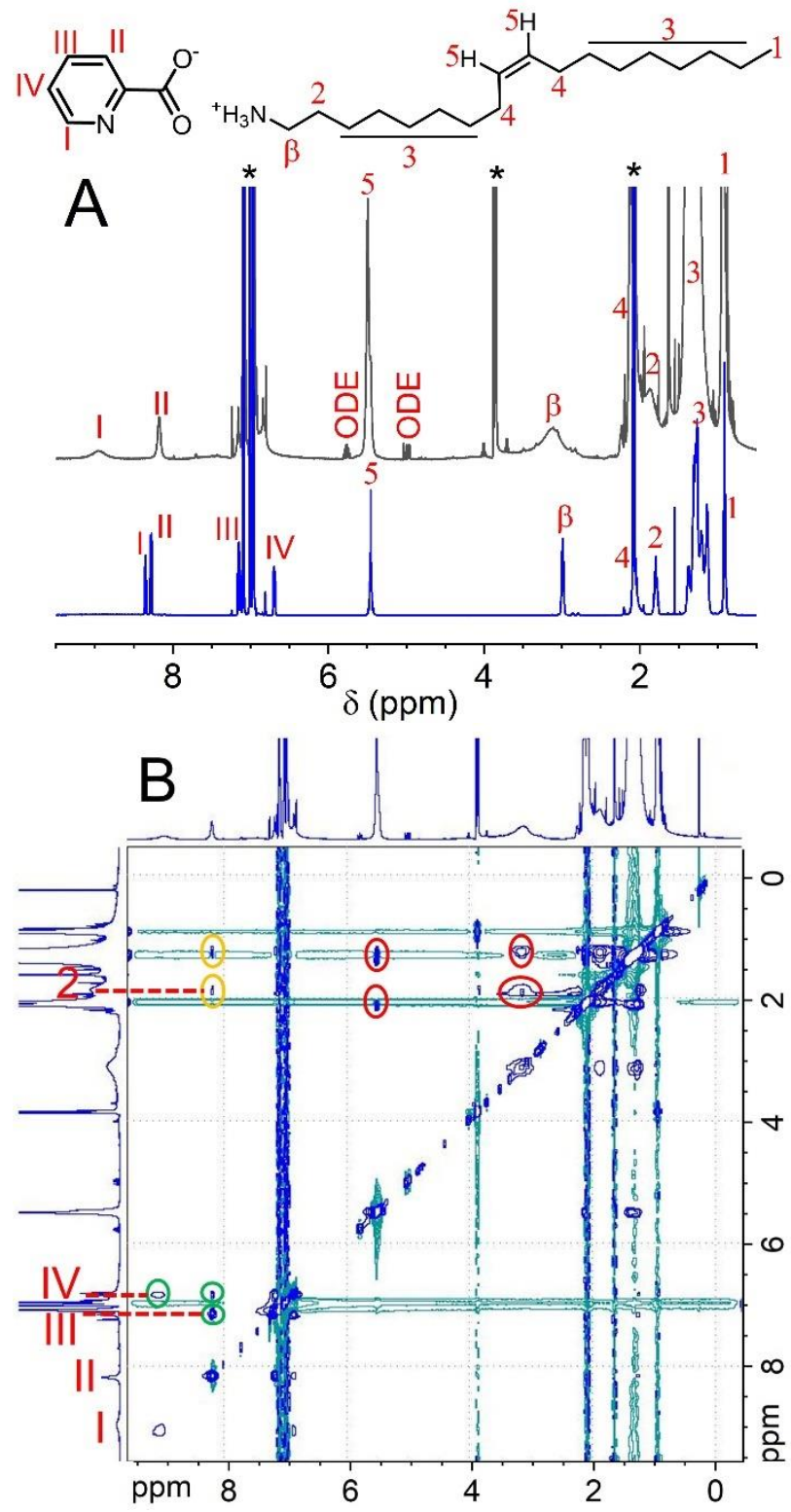

Figure 7. (A) $1 \mathrm{D}-{ }^{1} \mathrm{HNMR}$ spectra of the purified PNC with 200 equivalents addition of $\mathrm{OlAm}^{+} \mathrm{PIC}^{-}$ligands (Black) and the free $\mathrm{OlAm}^{+} \mathrm{PIC}^{-}$ligand (Blue) in toluene-D8. "**" marked signals belong to solvents. (B) 2D-NOESY of the PNC sample in (A). Critical off-diagonal cross-peaks featuring negative NOE are highlighted as red for oleylammonium protons, green for PIC protons, and yellow for OlAm/PIC intermolecular coupling. The magnified cross-peak regions can be found in the Supporting Information.

At last, we employed vibrational spectroscopy to characterize PIC bound on the PNC surface. In general, we notice a trend of hypsochromic shifts for vibrational patterns upon the bound to the PNC surface, as illustrated in Figure 8. Because of the coexistence of oleylammonium and residual oleate, we turn to DFT calculations to assist the assignment of 
vibrational bands of PIC, and the results are listed in Table S3. In line with the experimental spectra, when PIC is adsorbed to the PNC surface, most calculated vibrational modes show upshifted frequencies. It is known that the coordination of pyridine at the $\mathrm{N}$ atom as a Lewis base causes an increase in the overall bond strength of the aromatic ring, resulting in hypochromic shifts for the vibrational signals. ${ }^{75}$ The systematic vibrational frequency shift supports the participation of the pyridinic $\mathrm{N}$ atom in the coordination. In addition, vibrational frequencies observed for PIC on the PNC surface are similar to those of the $\mathrm{Ba}(\mathrm{PIC})_{2}$ complex, in which PIC behaves as a bidentate chelating ligand. ${ }^{76}$

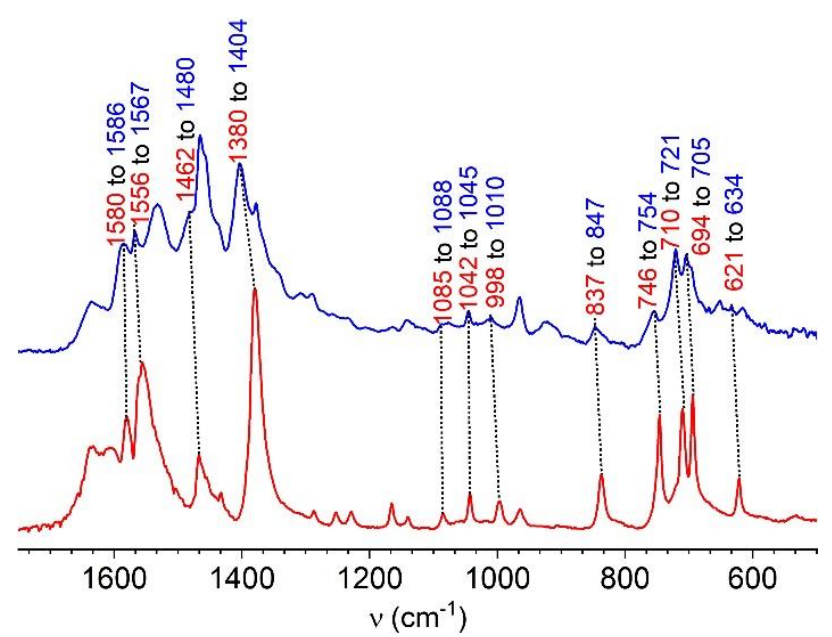

Figure 8. The comparison of the fingerprint region of FTIR spectra of purified PNCs (blue) and the pure $\mathrm{OlAm}^{+} \mathrm{PIC}^{-}$ligand (red). The notable frequency shifts upon binding are labeled above the spectrum of the purified PNC. Full spectra in between $400-3400 \mathrm{~cm}^{-1}$ are shown in Figure S14.

\section{CONCLUSIONS}

We demonstrate stable covalent interaction on the surface of PNC can be achieved through chelating coordination of a heterocyclic carboxylate, picolinate. With a bound affinity that is $15-30$ folds stronger than the original ligand, the PIC can effectively passivate the surface defect of PNCs in the strongly quantum confined regime in various solvents and bring the PLQY up to $>0.8$. The PL kinetics prove that the PIC passivation eliminates most of the surface traps and prolongs the exciton lifetimes in a subensemble of PNCs approaching the radiative 
relaxation limit. Combined NMR, IR, and DFT studies reveal the bidentate nature of PIC and the coadsorption of $\mathrm{OlAm}^{+} \mathrm{PIC}^{-}$ion pair on the PNC surface. In apolar solvents, the binding of PIC can approach stoichiometry, making PIC a good candidate of anchoring motifs to graft functional structures. The binding of PIC is strong enough to protect the small-size PNCs in polar solvents, including ethyl acetate. Based on the protective effect, we design a precipitation-redissolution purification procedure that can thoroughly remove the undesired synthetic residuals while maintaining strong quantum confinement in PNCs. We also demonstrate the potential of adapting this purification procedure to introduce new ammonium species to the PNC surface. The strong chelating coordination promises surface engineering that can modify the ligand shell of PNCs.

\section{ASSOCIATED CONTENT Supporting Information}

The Supporting Information is available free of charge on the website.

\section{AUTHOR INFORMATION Corresponding Author}

$*$

\section{ACKNOWLEDGEMENTS}

The authors would like to thank Queens College and the City University of New York for financial and facility support. The work is partly supported by the PSC-CUNY award. This research used resources of the National Energy Research Scientific Computing Center (NERSC), a U.S. Department of Energy Office of Science User Facility located at Lawrence Berkeley National Laboratory, operated under Contract No. DE-AC02-05CH11231.

\section{REFERENCES}

1. Weidman, M. C.; Goodman, A. J.; Tisdale, W. A., Colloidal Halide Perovskite Nanoplatelets: An Exciting New Class of Semiconductor Nanomaterials. Chem. Mater. 2017, 29 (12), 5019-5030. 
2. Akkerman, Q. A.; Rainò, G.; Kovalenko, M. V.; Manna, L., Genesis, challenges and opportunities for colloidal lead halide perovskite nanocrystals. Nat. Mater. 2018, 17 (5), 394-405. 3. Kovalenko, M. V.; Protesescu, L.; Bodnarchuk, M. I., Properties and potential optoelectronic applications of lead halide perovskite nanocrystals. Science 2017, 358 (6364), 745-750.

4. Schanze, K. S.; Kamat, P. V.; Yang, P.; Bisquert, J., Progress in Perovskite Photocatalysis. ACS Energy Lett. 2020, 5 (8), 2602-2604.

5. Dong, Y.; Qiao, T.; Kim, D.; Parobek, D.; Rossi, D.; Son, D. H., Precise Control of Quantum Confinement in Cesium Lead Halide Perovskite Quantum Dots via Thermodynamic Equilibrium. Nano Lett. 2018, 18 (6), 3716-3722.

6. $\quad$ Yang, D.; Zou, Y.; Li, P.; Liu, Q.; Wu, L.; Hu, H.; Xu, Y.; Sun, B.; Zhang, Q.; Lee, S.-T., Large-scale synthesis of ultrathin cesium lead bromide perovskite nanoplates with precisely tunable dimensions and their application in blue light-emitting diodes. Nano Energy 2018, 47, 235-242.

7. Akkerman, Q. A.; Motti, S. G.; Srimath Kandada, A. R.; Mosconi, E.; D'Innocenzo, V.; Bertoni, G.; Marras, S.; Kamino, B. A.; Miranda, L.; De Angelis, F., Solution Synthesis Approach to Colloidal Cesium Lead Halide Perovskite Nanoplatelets with Monolayer-Level Thickness Control. J. Am. Chem. Soc. 2016, 138, 1010.

8. Bekenstein, Y.; Koscher, B. A.; Eaton, S. W.; Yang, P. D.; Alivisatos, A. P., Highly Luminescent Colloidal Nanoplates of Perovskite Cesium Lead Halide and Their Oriented Assemblies. J. Am. Chem. Soc. 2015, 137, 16008.

9. Lv, L. F.; Xu, Y. B.; Fang, H. H.; Luo, W. J.; Xu, F. J.; Liu, L. M.; Wang, B. W.; Zhang, X. F.; Yang, D.; Hu, W. D., Generalized Colloidal Synthesis of High-Quality, Two-Dimensional Cesium Lead Halide Perovskite Nanosheets and Their Applications in Photodetectors. Nanoscale 2016, 8, 13589.

10. Nedelcu, G.; Protesescu, L.; Yakunin, S.; Bodnarchuk, M. I.; Grotevent, M. J.; Kovalenko, M. V., Fast Anion-Exchange in Highly Luminescent Nanocrystals of Cesium Lead Halide Perovskites $\left(\mathrm{CsPbX}_{3}, \mathrm{X}=\mathrm{Cl}, \mathrm{Br}, \mathrm{I}\right)$. Nano Lett. 2015, 15 (8), 5635-5640.

11. Ramasamy, P.; Lim, D.-H.; Kim, B.; Lee, S.-H.; Lee, M.-S.; Lee, J.-S., All-inorganic cesium lead halide perovskite nanocrystals for photodetector applications. Chem. Commun. 2016, 52 (10), 2067-2070.

12. Parobek, D.; Roman, B. J.; Dong, Y.; Jin, H.; Lee, E.; Sheldon, M.; Son, D. H., Excitonto-Dopant Energy Transfer in Mn-Doped Cesium Lead Halide Perovskite Nanocrystals. Nano Lett. 2016, 16, 7376.

13. Liu, M.; Zhong, G.; Yin, Y.; Miao, J.; Li, K.; Wang, C.; Xu, X.; Shen, C.; Meng, H., Aluminum-Doped Cesium Lead Bromide Perovskite Nanocrystals with Stable Blue Photoluminescence Used for Display Backlight. Adv. Sci. 2017, 4 (11), 1700335.

14. Yong, Z.-J.; Guo, S.-Q.; Ma, J.-P.; Zhang, J.-Y.; Li, Z.-Y.; Chen, Y.-M.; Zhang, B.-B.; Zhou, Y.; Shu, J.; Gu, J.-L.; Zheng, L.-R.; Bakr, O. M.; Sun, H.-T., Doping-Enhanced ShortRange Order of Perovskite Nanocrystals for Near-Unity Violet Luminescence Quantum Yield. $J$. Am. Chem. Soc. 2018, 140 (31), 9942-9951.

15. Pan, G.; Bai, X.; Yang, D.; Chen, X.; Jing, P.; Qu, S.; Zhang, L.; Zhou, D.; Zhu, J.; Xu, W.; Dong, B.; Song, H., Doping Lanthanide into Perovskite Nanocrystals: Highly Improved and Expanded Optical Properties. Nano Lett. 2017, 17 (12), 8005-8011.

16. Hines, D. A.; Kamat, P. V., Recent Advances in Quantum Dot Surface Chemistry. ACS Appl. Mater. Interf. 2014, 6 (5), 3041-3057. 
17. Weiss, E. A., Organic Molecules as Tools To Control the Growth, Surface Structure, and Redox Activity of Colloidal Quantum Dots. Acc. Chem. Res. 2013, 46 (11), 2607-2615.

18. Brown, P. R.; Kim, D.; Lunt, R. R.; Zhao, N.; Bawendi, M. G.; Grossman, J. C.; Bulovic, V., Energy Level Modification in Lead Sulfide Quantum Dot Thin Films through Ligand Exchange. ACS Nano 2014, 8 (6), 5863-5872.

19. Scheele, M.; Hanifi, D.; Zherebetskyy, D.; Chourou, S. T.; Axnanda, S.; Rancatore, B. J.; Thorkelsson, K.; Xu, T.; Liu, Z.; Wang, L.-W.; Liu, Y.; Alivisatos, A. P., PbS Nanoparticles Capped with Tetrathiafulvalenetetracarboxylate: Utilizing Energy Level Alignment for Efficient Carrier Transport. ACS Nano 2014, 8 (3), 2532-2540.

20. Kroupa, D. M.; Vörös, M.; Brawand, N. P.; McNichols, B. W.; Miller, E. M.; Gu, J.; Nozik, A. J.; Sellinger, A.; Galli, G.; Beard, M. C., Tuning colloidal quantum dot band edge positions through solution-phase surface chemistry modification. Nat. Comm. 2017, 8 (1), 15257. 21. Kroupa, D. M.; Vörös, M.; Brawand, N. P.; Bronstein, N.; McNichols, B. W.; Castaneda, C. V.; Nozik, A. J.; Sellinger, A.; Galli, G.; Beard, M. C., Optical Absorbance Enhancement in PbS QD/Cinnamate Ligand Complexes. J. Phys. Chem. Lett. 2018, 3425-3433.

22. Buckley, J. J.; Couderc, E.; Greaney, M. J.; Munteanu, J.; Riche, C. T.; Bradforth, S. E.; Brutchey, R. L., Chalcogenol Ligand Toolbox for CdSe Nanocrystals and Their Influence on Exciton Relaxation Pathways. ACS Nano 2014, 8 (3), 2512-2521.

23. He, C.; Weinberg, D. J.; Nepomnyashchii, A. B.; Lian, S.; Weiss, E. A., Control of the Redox Activity of PbS Quantum Dots by Tuning Electrostatic Interactions at the Quantum Dot/Solvent Interface. J. Am. Chem. Soc. 2016, 138 (28), 8847-8854.

24. Weinberg, D. J.; He, C.; Weiss, E. A., Control of the Redox Activity of Quantum Dots through Introduction of Fluoroalkanethiolates into Their Ligand Shells. J. Am. Chem. Soc. 2016, 138 (7), 2319-2326.

25. Kroupa, D. M.; Arias, D. H.; Blackburn, J. L.; Carroll, G. M.; Granger, D. B.; Anthony, J. E.; Beard, M. C.; Johnson, J. C., Control of Energy Flow Dynamics between Tetracene Ligands and $\mathrm{PbS}$ Quantum Dots by Size Tuning and Ligand Coverage. Nano Lett. 2018, 18 (2), 865-873.

26. Frederick, M. T.; Weiss, E. A., Relaxation of Exciton Confinement in CdSe Quantum Dots by Modification with a Conjugated Dithiocarbamate Ligand. Acs Nano 2010, 4 (6), 31953200 .

27. Lian, S.; Weinberg, D. J.; Harris, R. D.; Kodaimati, M. S.; Weiss, E. A., Subpicosecond Photoinduced Hole Transfer from a CdS Quantum Dot to a Molecular Acceptor Bound Through an Exciton-Delocalizing Ligand. ACS Nano 2016, 10 (6), 6372-6382.

28. Wang, C.; Kodaimati, M. S.; Schatz, G. C.; Weiss, E. A., The photoluminescence spectral profiles of water-soluble aggregates of $\mathrm{PbS}$ quantum dots assembled through reversible metal coordination. Chem. Commun. 2017.

29. Wang, C.; Weiss, E. A., Accelerating FRET between Near-Infrared Emitting Quantum Dots Using a Molecular J-Aggregate as an Exciton Bridge. Nano Lett. 2017, 17 (9), 5666-5671.

30. Mayilo, S.; Hilhorst, J.; Susha, A. S.; Hohl, C.; Franzl, T.; Klar, T. A.; Rogach, A. L.; Feldmann, J., Energy transfer in solution-based clusters of CdTe nanocrystals electrostatically bound by calcium ions. J. Phys. Chem. C 2008, 112 (37), 14589-14594.

31. Halpert, J. E.; Tischler, J. R.; Nair, G.; Walker, B. J.; Liu, W.; Bulovic, V.; Bawendi, M. G., Electrostatic Formation of Quantum Dot/J-aggregate FRET Pairs in Solution. J. Phys. Chem. C 2009, 113 (23), 9986-9992. 
32. Zhang, Q.; Atay, T.; Tischler, J. R.; Bradley, M. S.; Bulovic, V.; Nurmikko, A. V., Highly efficient resonant coupling of optical excitations in hybrid organic/inorganic semiconductor nanostructures. Nat Nano 2007, 2 (9), 555-559.

33. Qiao, Y.; Polzer, F.; Kirmse, H.; Steeg, E.; Kühn, S.; Friede, S.; Kirstein, S.; Rabe, J. P., Nanotubular J-Aggregates and Quantum Dots Coupled for Efficient Resonance Excitation Energy Transfer. ACS Nano 2015, 9 (2), 1552-1560.

34. Howes, P. D.; Chandrawati, R.; Stevens, M. M., Colloidal nanoparticles as advanced biological sensors. Science 2014, 346 (6205).

35. Zhang, Y.; Wang, T.-H., Quantum Dot Enabled Molecular Sensing and Diagnostics. Theranostics 2012, 2 (7), 631-654.

36. Wu, Y.; Eisele, K.; Doroshenko, M.; Algara-Siller, G.; Kaiser, U.; Koynov, K.; Weil, T., Quantum Dots: A Quantum Dot Photoswitch for DNA Detection, Gene Transfection, and LiveCell Imaging (Small 22/2012). Small 2012, 8 (22), 3381-3381.

37. De Roo, J.; Ibáñez, M.; Geiregat, P.; Nedelcu, G.; Walravens, W.; Maes, J.; Martins, J. C.; Van Driessche, I.; Kovalenko, M. V.; Hens, Z., Highly Dynamic Ligand Binding and Light Absorption Coefficient of Cesium Lead Bromide Perovskite Nanocrystals. ACS Nano 2016, 10 (2), 2071-2081.

38. Smock, S. R.; Williams, T. J.; Brutchey, R. L., Quantifying the Thermodynamics of Ligand Binding to $\mathrm{CsPbBr}_{3}$ Quantum Dots. Angew. Chem., Int. Ed. 2018, 57 (36), 11711-11715.

39. Smock, S. R.; Chen, Y.; Rossini, A. J.; Brutchey, R. L., The Surface Chemistry and Structure of Colloidal Lead Halide Perovskite Nanocrystals. Acc. Chem. Res. 2021, 54 (3), 707718.

40. Pradhan, N., Alkylammonium Halides for Facet Reconstruction and Shape Modulation in Lead Halide Perovskite Nanocrystals. Acc. Chem. Res. 2021, 54 (5), 1200-1208.

41. Toso, S.; Baranov, D.; Manna, L., Metamorphoses of Cesium Lead Halide Nanocrystals. Acc. Chem. Res. 2021, 54 (3), 498-508.

42. Kang, J.; Wang, L.-W., High Defect Tolerance in Lead Halide Perovskite $\mathrm{CsPbBr}_{3} . J$. Phys. Chem. Lett. 2017, 8 (2), 489-493.

43. Shi, H.; Du, M.-H., Shallow halogen vacancies in halide optoelectronic materials. Phys. Rev. B 2014, 90 (17), 174103.

44. Ravi, V. K.; Swarnkar, A.; Chakraborty, R.; Nag, A., Excellent green but less impressive blue luminescence from $\mathrm{CsPbBr}_{3}$ Perovskite Nanocubes and Nanoplatelets. Nanotechnology 2016, 27, 325708 .

45. Hoshi, K.; Chiba, T.; Sato, J.; Hayashi, Y.; Takahashi, Y.; Ebe, H.; Ohisa, S.; Kido, J., Purification of Perovskite Quantum Dots Using Low-Dielectric-Constant Washing Solvent "Diglyme" for Highly Efficient Light-Emitting Devices. ACS Appl. Mater. Interf. 2018, 10 (29), 24607-24612.

46. Kim, Y.; Yassitepe, E.; Voznyy, O.; Comin, R.; Walters, G.; Gong, X.; Kanjanaboos, P.; Nogueira, A. F.; Sargent, E. H., Efficient Luminescence from Perovskite Quantum Dot Solids. ACS Appl. Mater. Interf. 2015, 7 (45), 25007-25013.

47. Nenon, D. P.; Pressler, K.; Kang, J.; Koscher, B. A.; Olshansky, J. H.; Osowiecki, W. T.; Koc, M. A.; Wang, L.-W.; Alivisatos, A. P., Design Principles for Trap-Free $\mathrm{CsPbX}_{3}$ Nanocrystals: Enumerating and Eliminating Surface Halide Vacancies with Softer Lewis Bases. J. Am. Chem. Soc. 2018, 140 (50), 17760-17772. 
48. Koscher, B. A.; Swabeck, J. K.; Bronstein, N. D.; Alivisatos, A. P., Essentially Trap-Free $\mathrm{CsPbBr}_{3}$ Colloidal Nanocrystals by Postsynthetic Thiocyanate Surface Treatment. J. Am. Chem. Soc. 2017, 139 (19), 6566-6569.

49. Zhong, Q.; Cao, M.; Xu, Y.; Li, P.; Zhang, Y.; Hu, H.; Yang, D.; Xu, Y.; Wang, L.; Li,

Y.; Zhang, X.; Zhang, Q., L-Type Ligand-Assisted Acid-Free Synthesis of $\mathrm{CsPbBr}_{3}$ Nanocrystals with Near-Unity Photoluminescence Quantum Yield and High Stability. Nano Lett. 2019, 19 (6), 4151-4157.

50. $\quad$ Krieg, F.; Ochsenbein, S. T.; Yakunin, S.; ten Brinck, S.; Aellen, P.; Süess, A.; Clerc, B.; Guggisberg, D.; Nazarenko, O.; Shynkarenko, Y.; Kumar, S.; Shih, C.-J.; Infante, I.; Kovalenko, M. V., Colloidal $\mathrm{CsPbX}_{3}(\mathrm{X}=\mathrm{Cl}, \mathrm{Br}, \mathrm{I})$ Nanocrystals 2.0: Zwitterionic Capping Ligands for Improved Durability and Stability. ACS Energy Lett. 2018, 3 (3), 641-646.

51. Yang, D.; Li, X.; Zhou, W.; Zhang, S.; Meng, C.; Wu, Y.; Wang, Y.; Zeng, H., CsPbBr 3 Quantum Dots 2.0: Benzenesulfonic Acid Equivalent Ligand Awakens Complete Purification. Adv. Mater. 2019, 31 (30), 1900767.

52. $\quad$ Pan, J.; Quan, L. N.; Zhao, Y.; Peng, W.; Murali, B.; Sarmah, S. P.; Yuan, M.; Sinatra, L.; Alyami, N. M.; Liu, J.; Yassitepe, E.; Yang, Z.; Voznyy, O.; Comin, R.; Hedhili, M. N.; Mohammed, O. F.; Lu, Z. H.; Kim, D. H.; Sargent, E. H.; Bakr, O. M., Highly Efficient Perovskite-Quantum-Dot Light-Emitting Diodes by Surface Engineering. Adv. Mater. 2016, 28 (39), 8718-8725.

53. Khan, J.; Zhang, X.; Yuan, J.; Wang, Y.; Shi, G.; Patterson, R.; Shi, J.; Ling, X.; Hu, L.; Wu, T.; Dai, S.; Ma, W., Tuning the Surface-Passivating Ligand Anchoring Position Enables Phase Robustness in $\mathrm{CsPbI}_{3}$ Perovskite Quantum Dot Solar Cells. ACS Energy Lett. 2020, 5 (10), 3322-3329.

54. Pan, J.; Shang, Y.; Yin, J.; De Bastiani, M.; Peng, W.; Dursun, I.; Sinatra, L.; El-Zohry, A. M.; Hedhili, M. N.; Emwas, A.-H.; Mohammed, O. F.; Ning, Z.; Bakr, O. M., Bidentate Ligand-Passivated $\mathrm{CsPbI}_{3}$ Perovskite Nanocrystals for Stable Near-Unity Photoluminescence Quantum Yield and Efficient Red Light-Emitting Diodes. J. Am. Chem. Soc. 2018, 140 (2), $562-$ 565.

55. Bodnarchuk, M. I.; Boehme, S. C.; ten Brinck, S.; Bernasconi, C.; Shynkarenko, Y.; Krieg, F.; Widmer, R.; Aeschlimann, B.; Günther, D.; Kovalenko, M. V.; Infante, I., Rationalizing and Controlling the Surface Structure and Electronic Passivation of Cesium Lead Halide Nanocrystals. ACS Energy Lett. 2019, 4 (1), 63-74.

56. Imran, M.; Ijaz, P.; Goldoni, L.; Maggioni, D.; Petralanda, U.; Prato, M.; Almeida, G.; Infante, I.; Manna, L., Simultaneous Cationic and Anionic Ligand Exchange For Colloidally Stable CsPbBr 3 Nanocrystals. ACS Energy Lett. 2019, 4 (4), 819-824.

57. DuBose, J. T.; Kamat, P. V., Surface Chemistry Matters. How Ligands Influence Excited State Interactions between $\mathrm{CsPbBr}_{3}$ and Methyl Viologen. J. Phys. Chem. C 2020, 124 (24), 12990-12998.

58. Vickers, E. T.; Enlow, E. E.; Delmas, W. G.; DiBenedetto, A. C.; Chowdhury, A. H.; Bahrami, B.; Dreskin, B. W.; Graham, T. A.; Hernandez, I. N.; Carter, S. A.; Ghosh, S.; Qiao, Q.; Zhang, J. Z., Enhancing Charge Carrier Delocalization in Perovskite Quantum Dot Solids with Energetically Aligned Conjugated Capping Ligands. ACS Energy Lett. 2020, 5 (3), 817-825.

59. Li, Y.; Shu, Q.; Du, Q.; Dai, Y.; Zhao, S.; Zhang, J.; Li, L.; Chen, K., Surface Modification for Improving the Photocatalytic Polymerization of 3,4-Ethylenedioxythiophene over Inorganic Lead Halide Perovskite Quantum Dots. ACS Appl. Mater. Interf. 2020, 12 (1), 451-460. 
60. Noel, N. K.; Abate, A.; Stranks, S. D.; Parrott, E. S.; Burlakov, V. M.; Goriely, A.; Snaith, H. J., Enhanced Photoluminescence and Solar Cell Performance via Lewis Base Passivation of Organic-Inorganic Lead Halide Perovskites. ACS Nano 2014, 8 (10), 9815-9821.

61. Maes, J.; Balcaen, L.; Drijvers, E.; Zhao, Q.; De Roo, J.; Vantomme, A.; Vanhaecke, F.; Geiregat, P.; Hens, Z., Light Absorption Coefficient of $\mathrm{CsPBr}_{3}$ Perovskite Nanocrystals. J. Phys. Chem. Lett. 2018, 9 (11), 3093-3097.

62. Bohn, B. J.; Tong, Y.; Gramlich, M.; Lai, M. L.; Döblinger, M.; Wang, K.; Hoye, R. L. Z.; Müller-Buschbaum, P.; Stranks, S. D.; Urban, A. S.; Polavarapu, L.; Feldmann, J., Boosting Tunable Blue Luminescence of Halide Perovskite Nanoplatelets through Postsynthetic Surface Trap Repair. Nano Lett. 2018, 18 (8), 5231-5238.

63. Ebenstein, Y.; Mokari, T.; Banin, U., Fluorescence quantum yield of CdSe/ZnS nanocrystals investigated by correlated atomic-force and single-particle fluorescence microscopy. Appl. Phys. Lett. 2002, 80 (21), 4033-4035.

64. Wehrenberg, B. L.; Wang, C.; Guyot-Sionnest, P., Interband and Intraband Optical Studies of PbSe Colloidal Quantum Dots. J. Phys. Chem. B 2002, 106 (41), 10634-10640.

65. Hens, Z.; Moreels, I., Light absorption by colloidal semiconductor quantum dots. $J$. Mater. Chem. 2012, 22 (21), 10406-10415.

66. Yan, W.; Mao, L.; Zhao, P.; Mertens, A.; Dottermusch, S.; Hu, H.; Jin, Z.; Richards, B. S., Determination of complex optical constants and photovoltaic device design of all-inorganic $\mathrm{CsPbBr}_{3}$ perovskite thin films. Opt. Express 2020, 28 (10), 15706-15717.

67. Marbella, L. E.; Millstone, J. E., NMR Techniques for Noble Metal Nanoparticles. Chem. Mater. 2015, 27 (8), 2721-2739.

68. Hens, Z.; Martins, J. C., A Solution NMR Toolbox for Characterizing the Surface Chemistry of Colloidal Nanocrystals. Chem. Mater. 2013, 25 (8), 1211-1221.

69. Harris, R. D.; Amin, V. A.; Lau, B.; Weiss, E. A., Role of Interligand Coupling in Determining the Interfacial Electronic Structure of Colloidal CdS Quantum Dots. ACS Nano 2016, 10 (1), 1395-1403.

70. Chen, K.; Zhong, Q.; Chen, W.; Sang, B.; Wang, Y.; Yang, T.; Liu, Y.; Zhang, Y.; Zhang, H., Short-Chain Ligand-Passivated Stable $\alpha-\mathrm{CsPbI}_{3}$ Quantum Dot for All-Inorganic Perovskite Solar Cells. Adv. Func. Mater. 2019, 29 (24), 1900991.

71. Thompson, N. J.; Wilson, M. W. B.; Congreve, D. N.; Brown, P. R.; Scherer, J. M.; Bischof, T. S.; Wu, M.; Geva, N.; Welborn, M.; Voorhis, T. V.; Bulović, V.; Bawendi, M. G.; Baldo, M. A., Energy harvesting of non-emissive triplet excitons in tetracene by emissive $\mathrm{PbS}$ nanocrystals. Nat Mater 2014, 13 (11), 1039-1043.

72. Zhang, Z.; Edme, K.; Lian, S.; Weiss, E. A., Enhancing the Rate of Quantum-DotPhotocatalyzed Carbon-Carbon Coupling by Tuning the Composition of the Dot's Ligand Shell. J. Am. Chem. Soc. 2017, 139 (12), 4246-4249.

73. Gao, Y.; Shi, E.; Deng, S.; Shiring, S. B.; Snaider, J. M.; Liang, C.; Yuan, B.; Song, R.; Janke, S. M.; Liebman-Peláez, A.; Yoo, P.; Zeller, M.; Boudouris, B. W.; Liao, P.; Zhu, C.; Blum, V.; Yu, Y.; Savoie, B. M.; Huang, L.; Dou, L., Molecular engineering of organicinorganic hybrid perovskites quantum wells. Nat. Chem. 2019, 11 (12), 1151-1157.

74. Xue, J.; Wang, R.; Chen, X.; Yao, C.; Jin, X.; Wang, K.-L.; Huang, W.; Huang, T.; Zhao, Y.; Zhai, Y.; Meng, D.; Tan, S.; Liu, R.; Wang, Z.-K.; Zhu, C.; Zhu, K.; Beard, M. C.; Yan, Y.; Yang, Y., Reconfiguring the band-edge states of photovoltaic perovskites by conjugated organic cations. Science 2021, 371 (6529), 636-640. 
75. Ferwerda, R.; van der Maas, J. H.; van Duijneveldt, F. B., Pyridine adsorption onto metal oxides: an ab initio study of model systems. J. Mol. Catal. A Chem. 1996, 104 (3), 319-328.

76. Świderski, G.; Kalinowska, M.; Wojtulewski, S.; Lewandowski, W., Experimental (FTIR, FT-Raman, ${ }^{1} \mathrm{H}$ NMR) and theoretical study of magnesium, calcium, strontium, and barium picolinates. Spectrochim. Acta A Mol. Biomol. Spectrosc. 2006, 64 (1), 24-33. 